\title{
CONDITIONS AND CHARACTERISTICS OF WATER CRYSTALLIZATION ON THE WORKING SURFACE OF EVAPORATOR HEAT PUMPS IN RESERVOIRS WITH LOW TEMPERATURES
}

\author{
Vyacheslav I. Maksimov, Tatiana A. Nagornova, Vladimir S. Chernyshev \\ Institute of Power Engineering, National research Tomsk Polytechnic University, Tomsk 634050, \\ Russia
}

\begin{abstract}
Is carried out the mathematical simulation of heat transfer processes in the small environment of vaporizer camera of heat pump (HP) when the source of lowpotential energy is present. Are obtained the temperature distributions, which characterize thermal condition HP. Is carried out the comparative analysis of the results of mathematical simulation and experimental data from the work of heat-pumping installation in the conditions of relatively low (up to $4{ }^{\circ} \mathrm{C}$ ) temperatures. In the experiments, the partial freezing around of the tubes of vaporizer is established. It is revealed, that the formation of the layer of ice on the surface of vaporizer leads to the temperature contrast by the volume of liquid in the camera and reduction in the effectiveness in the work of heat-pumping installation.
\end{abstract}

\section{Introduction}

The efficiency of the heat pump (HP) depends on several factors [1]. The surface condition of vaporizer is one of them. In the case of its icing the heat-transfer, intensity of low-potential energy to heat-transfer agent is reduced. In the case of its icing the heat-transfer intensity of low-potential energy to heat-transfer agent is reduced. Therefore, the ranges of operating temperatures most common HP with the evaporative elements in the reservoirs with the relatively low temperature are substantially limited. But, from other side, the expansion of these ranges at least on several degrees can lead to the significant positive results in the work HP. Therefore the analysis of conditions and characteristics of the process of crystallizing the water on the working surfaces of the vaporizers of the heat pumps, which work in the reservoirs, is urgent task. Up to now studies of the processes of heat transfer in surrounding water evaporator were not conducted.

\section{Statement of the Problem}

In general, there are two basic modes of heat transfer between the cold water and the surface of the evaporator - free (in closed reservoirs) and mixed (with an ordered movement of water) convection. There are models that describe the processes of fluid flow and heat transfer in closed areas to conducted with local sources of heat release [2,3] in a mixed [4] convection (also with local energy 
supply in one of the boundaries of modeling). Models of [2-4] have been developed for conditions of intense local radiant heating [5.6] in the accumulation of energy in the building envelopes. Approach [2-6] is used for solving the problem of heat transfer in the evaporator HP (Fig. 1).

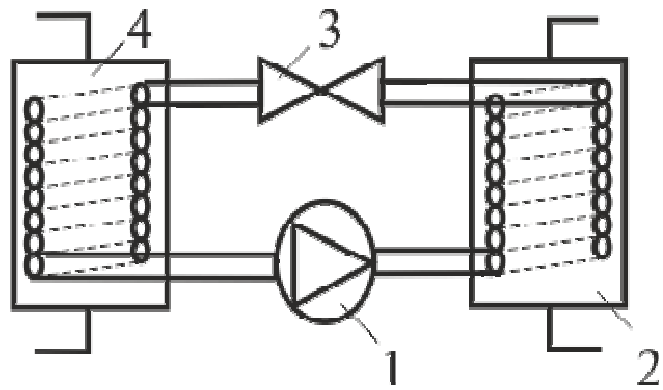

Figure 1. The experimental heat pump system: 1 - compressor, 2 - capacitor 3 - expansion valve, 4 - evaporator

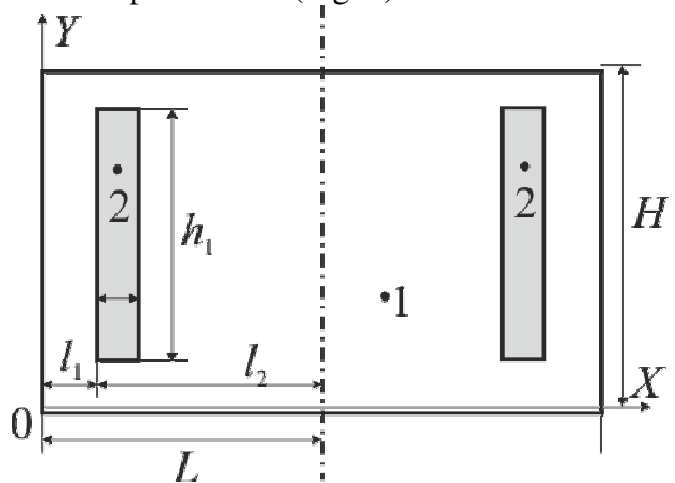

Figure 2. Scope of the task: 1 - the liquid in the evaporator; 2 - tube evaporator; $L, H$ - the length and width of the solutions.

We solved the problem of axisymmetric convective heat transfer for a rectangular cavity with a heat exchanger-evaporator of the heat pump (Fig. 2).

Navier-Stokes equations and the energy of the water and the evaporator in this case is:

$$
\begin{gathered}
\frac{\partial \Omega}{\partial \tau}+U \frac{\partial \Omega}{\partial X}+V \frac{\partial \Omega}{\partial Y}=\frac{1}{\sqrt{\mathrm{Gr}}} \Delta \Omega+\frac{1}{2} \frac{\partial \Theta}{\partial X}, \\
\frac{\partial \Theta}{\partial \tau}+U \frac{\partial \Theta}{\partial X}+V \frac{\partial \Theta}{\partial Y}=\frac{1}{\operatorname{Pr} \sqrt{\mathrm{Gr}}} \Delta \Theta \\
\frac{\partial^{2} \Psi}{\partial X^{2}}+\frac{\partial^{2} \Psi}{\partial Y^{2}}=\Omega .
\end{gathered}
$$

here $X, Y$ - dimensionless Cartesian coordinates; $\tau$ - dimensionless time; $\mathrm{Gr}=\mathrm{g} \beta \mathrm{L}^{3} \Delta T / v^{2}-$ Grashof number; g- acceleration produced by the mass forces, $\mathrm{m} / \mathrm{s}^{2} ; \beta$ - temperature coefficient of volume expansion, $\mathrm{K}^{-1} ; v$ - kinematic viscosity of the liquid; $L$ - characteristic size of the cavity; $\operatorname{Pr}=v / a-$ Prandtl number; $a-$ thermal diffusivity, $\mathrm{m}^{2} / \mathrm{s} ; \Theta=\left(T-T_{0}\right) /\left(T_{i t}-T_{0}\right)-$ dimensionless temperature; $T$ - temperature, $\mathrm{K} ; T_{0}$ - temperature at the initial time, $\mathrm{K} ; T_{i t}$ - scale of temperature, $\mathrm{K}$; $\Psi$-dimensionless stream function; $\Omega$-dimensionless vorticity; $\tau$-dimensionless time;

The initial conditions:

$$
\begin{aligned}
& \Psi(X, Y, 0)=0, \\
& \Omega(X, Y, 0)=0, \\
& \Theta(X, Y, 0)=0
\end{aligned}
$$

Boundary conditions:

- three external borders of the area set the conditions of insulation:

$$
\frac{\partial \Theta(X, Y, \tau)}{\partial n}=0
$$

- on the boundary $\mathrm{X}=\mathrm{L}$ given symmetry conditions:

$$
\frac{\partial^{2} \Theta(X, Y, \tau)}{\partial n^{2}}=0, \frac{\partial^{2} \Psi}{\partial n^{2}}=0 .
$$

- at the boundaries of the heat exchanger: 


$$
\Psi=0, \frac{\partial \Psi}{\partial X(\partial Y)}=0, \frac{\partial \Theta(X, Y, \tau)}{\partial n}=K i
$$

here $\mathrm{Ki}=q L^{3} / \lambda\left(T_{i t}-T_{0}\right)-$ number of Kirpichev; $\lambda-$ the thermal conductivity of a solid wall, $\mathrm{W} /(\mathrm{m} \cdot \mathrm{K})$.

The peculiarity of the problem being solved is to form on the evaporator surface layer of ice (crystallized water) has a significant impact on the conditions of the heat supply to the evaporator. Analysis of methods, algorithms and results of solving similar problems of heat transfer in the conditions of intensive phase transformations [7] showed that the modeling of intense absorption heat (evaporation) leads to a significant complication of procedures and algorithms, as well as to increase the duration of the repeated calculations. Similar problems should arise in the solution of problems of local intense heat (in the conditions of the condensation). For these reasons, when solving the problem stated above the effect of heat during the crystallization of water on the surface of the evaporator TN was ignored. The validity of this assumption was also confirmed by evaluation of convection, and conduction heat fluxes due to the crystallization of the surface of the evaporator using the machine [8].

The numerical solution of the problem (1) - (7) carried out by finite difference method using an algorithm for solving a proven group of tasks $[2,3,5,6]$ conjugate heat transfer in areas with local sources of energy. To substantiate the reliability of the results of numerical modeling audited conservative difference scheme is similar $[9,10]$.

\section{Simulation results}

Figure 3 shows the line current and temperature field, characterizing the convective heat transfer in a viscous incompressible fluid surrounding the evaporator of the heat pump. It was established that two of the central vortex formed: in the lower and upper parts of the rectangular area under consideration. At the initial time $(1000 s)$ is well defined temperature inhomogeneity in the depth of the tank and there is a possibility of freezing of the heat exchanger. Cold water mass flow circulating about the heat is not mixed with the upper main fluid flow. As a result, a dead space, which reduces the heat transfer rate of the evaporator with the ambient liquid. When time is reached $15000 \mathrm{~s}$ with the temperature field in the cross section $X=L$ becomes almost uniform height.

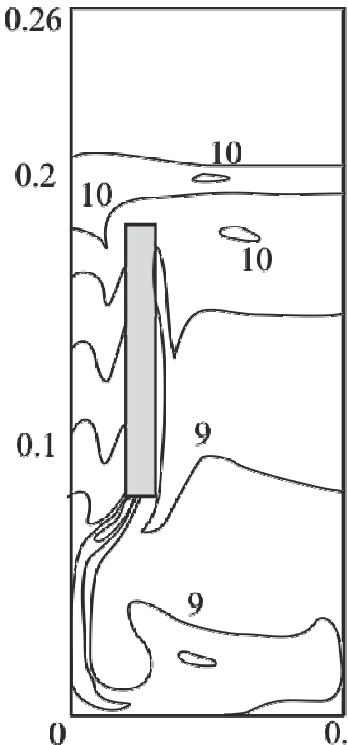

$a$

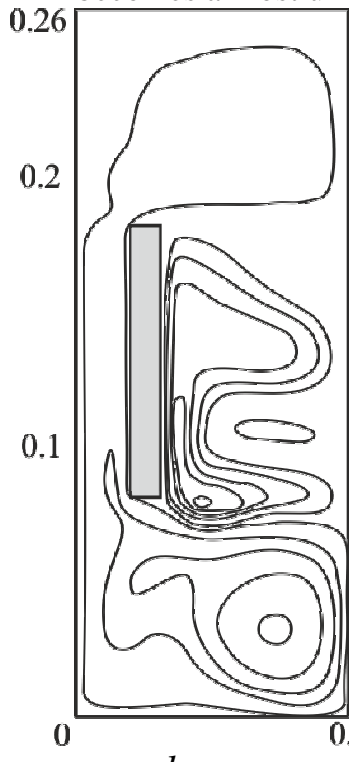

$b$

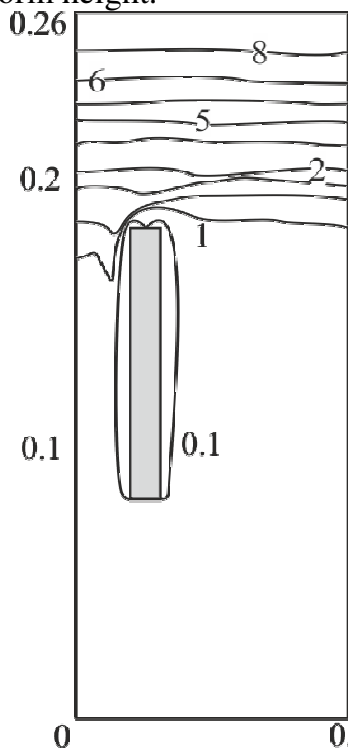

$c$

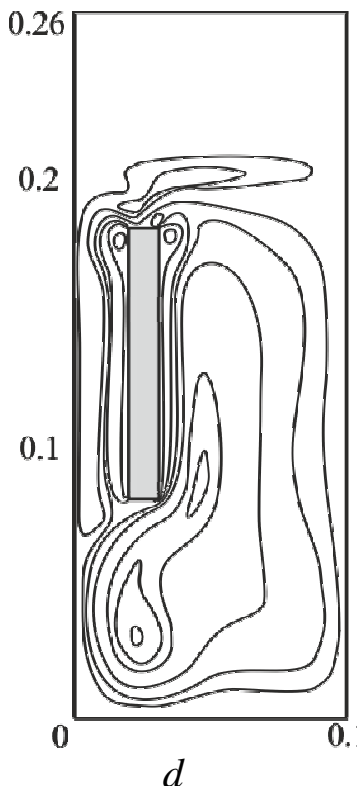

$d$

Figure 3. Temperature field $(a, b)$ and the contour of the stream function $(c, d)$ for various $\tau$ : 


$$
(a, b)-\tau=1000 \mathrm{c} ;(c, d)-\tau=15000 \mathrm{c} .
$$

\section{The methodology of experimental studies}

Experimental studies were carried out using the apparatus (Fig. 1), which is a vapor compression heat pump with its dedicated camera (dimensions $0,20 \times 0,25 \times 0,26 \mathrm{~m}$ ), the evaporator heat exchanger. As a source of low-grade heat is used with fresh water close to the real conditions of water bodies in regions with cold climates temperature $\left(4{ }^{\circ} \mathrm{C}<T<14{ }^{\circ} \mathrm{C}\right)$. The experimental setup includes a compressor, an expansion valve, two heat exchangers (evaporator, condenser) and test equipment (Fig. 1).

In the development of the experimental procedure were the main tasks of ensuring the conditions of temperature measurement on the surface of the evaporator in the HP and small neighborhood. At the same time we take into account the conclusions [11], justifying the use of thermocouples to measure the temperature of the medium under the conditions of natural convection in confined areas with local sources of energy. The temperature of the low-potential power in the chamber, the evaporator in the experiments was $14{ }^{\circ} \mathrm{C}$; ambient temperature $-\mathrm{T}_{o s}=20{ }^{\circ} \mathrm{C}$. The volume of water in the tank in the series of experiments was $0.016 \mathrm{~m}^{3}$.

Temperature measurement was carried out on the surfaces of heat exchanger tubes (see Fig. 5.6) and in specific points in space (in section $X=L / 2$ ) inside the pilot block (Fig. 7, 8) filled with water using graded chromel-alumel thermocouple junction diameter of $1 \mathrm{~mm}$. To determine the errors of measurement results each experiment was repeated several times at the fixed initial data and external conditions. Measurements were carried out simultaneously by 4 blocks of 8 thermocouples each.

In the experiment, a thermocouple fixed in predetermined time intervals: first hour every 5 minutes, then for three hours every 20 minutes.

To connect an analog-to-digital converter with a personal computer, the adapter uses a network that provides galvanic isolation between devices. As a means of data processing software package used by LabVIEW. The values of temperatures were recorded in the real time with the retention of the obtained results of measurements. The total relative error of determining the values of temperature did not exceed $4 \%$ in the whole range of parameters.

\section{The experimental results and discussion}

In Fig. 4,5 are represented typical values of local temperatures, conducting of experimental studies obtained as a result.

Distributions $T(\tau)$ in Fig. 4 is characterized heat exchange between the heat-transfer agent and the heated liquid, whose intensity considerably was reduced after 9000 seconds of the work of heatpumping installation. It should be noted that from 1200 second begins the freezing around of the upper tube of vaporizer (Fig. 5). Reduction in the temperature in the lower part of the vaporizer (Fig. 5 ) it is noticeable to 10000 seconds, after which the rate of crystallization slows down also to the end of the experiment practically does not change (Fig. 6,7).

The results of mathematical modeling of free-convective flows in a closed region in the presence of heat source are presented in Fig. 2-6. Numerical studies were conducted at the temperature: initial $\Theta_{0}=0$, the heat source - $\Theta i t=1$, the environment $-\Theta_{e}=0$. 
TSOTR 2015

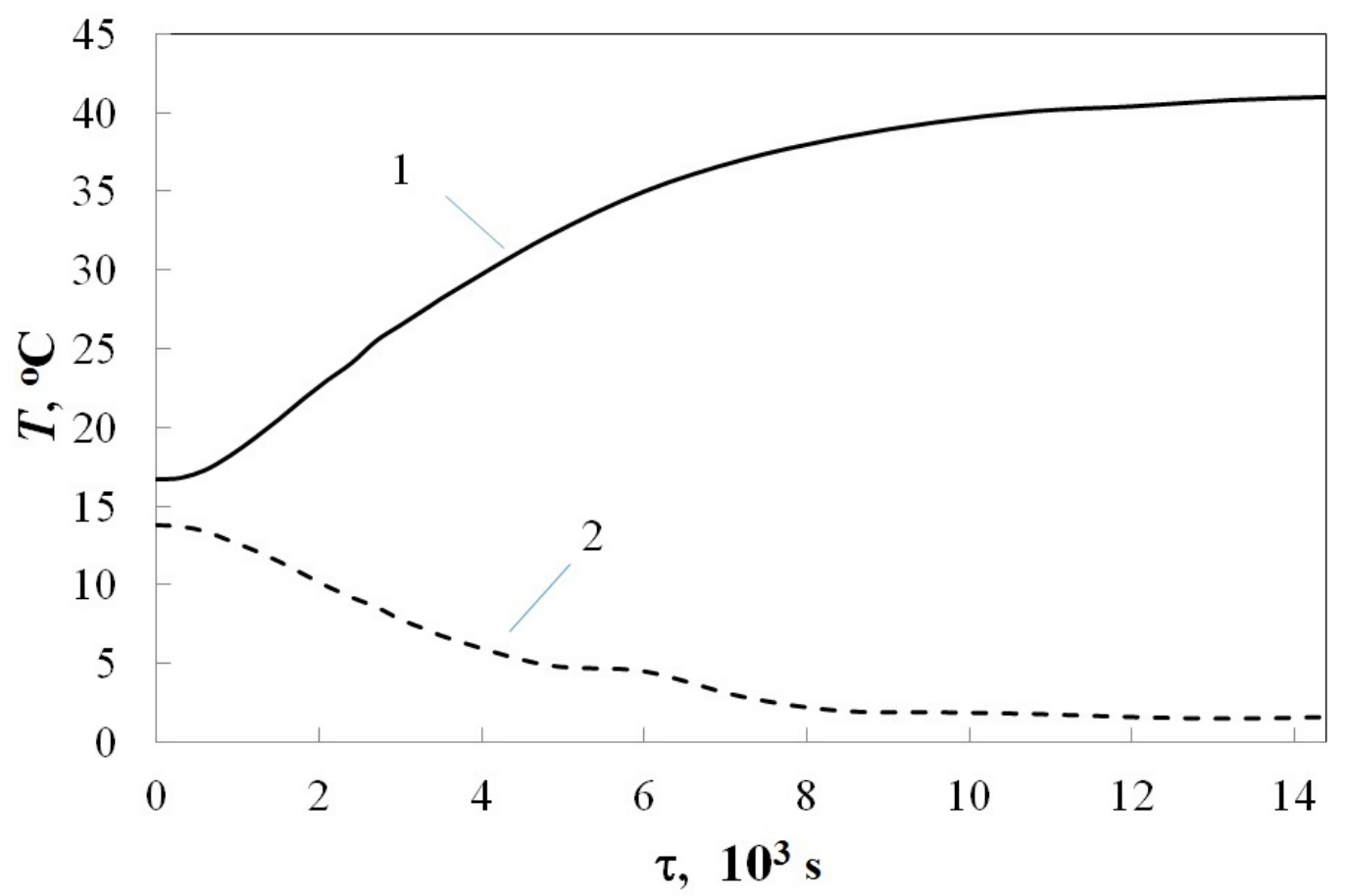

Figure 4. The change in temperature of the liquid (1) and the carrier (2) in time

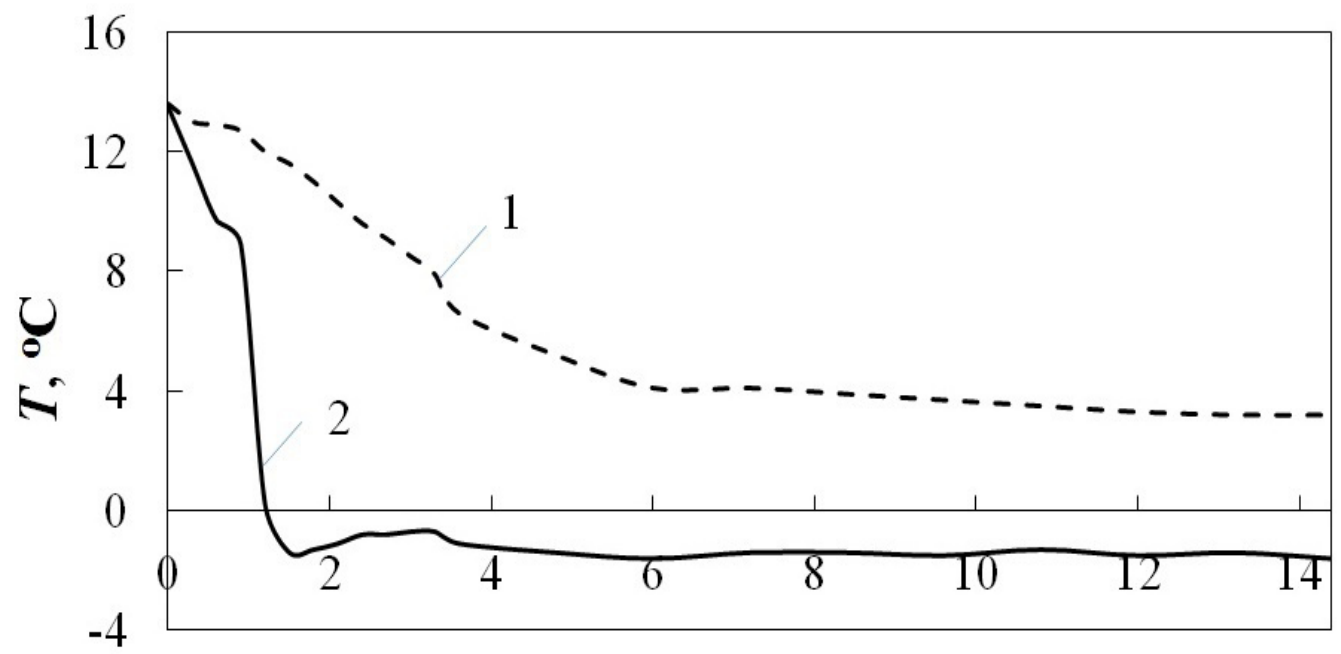

\section{$\tau, 10^{3} \mathrm{~s}$}

Figure 5. Temperature change of surfaces of the lower (1) and upper (2) of the evaporator tubes with the passage of time 
In the initial period of time rate of icing is high (Fig. 6,7). This is due to the fact that there is intense heat transfer from the low-potential power source to the wall of the heat exchanger. But the ice cover reduces heat transfer in the evaporator and after $6000 s$ to work HP ice thickness reaches its maximum value (Figure 7).

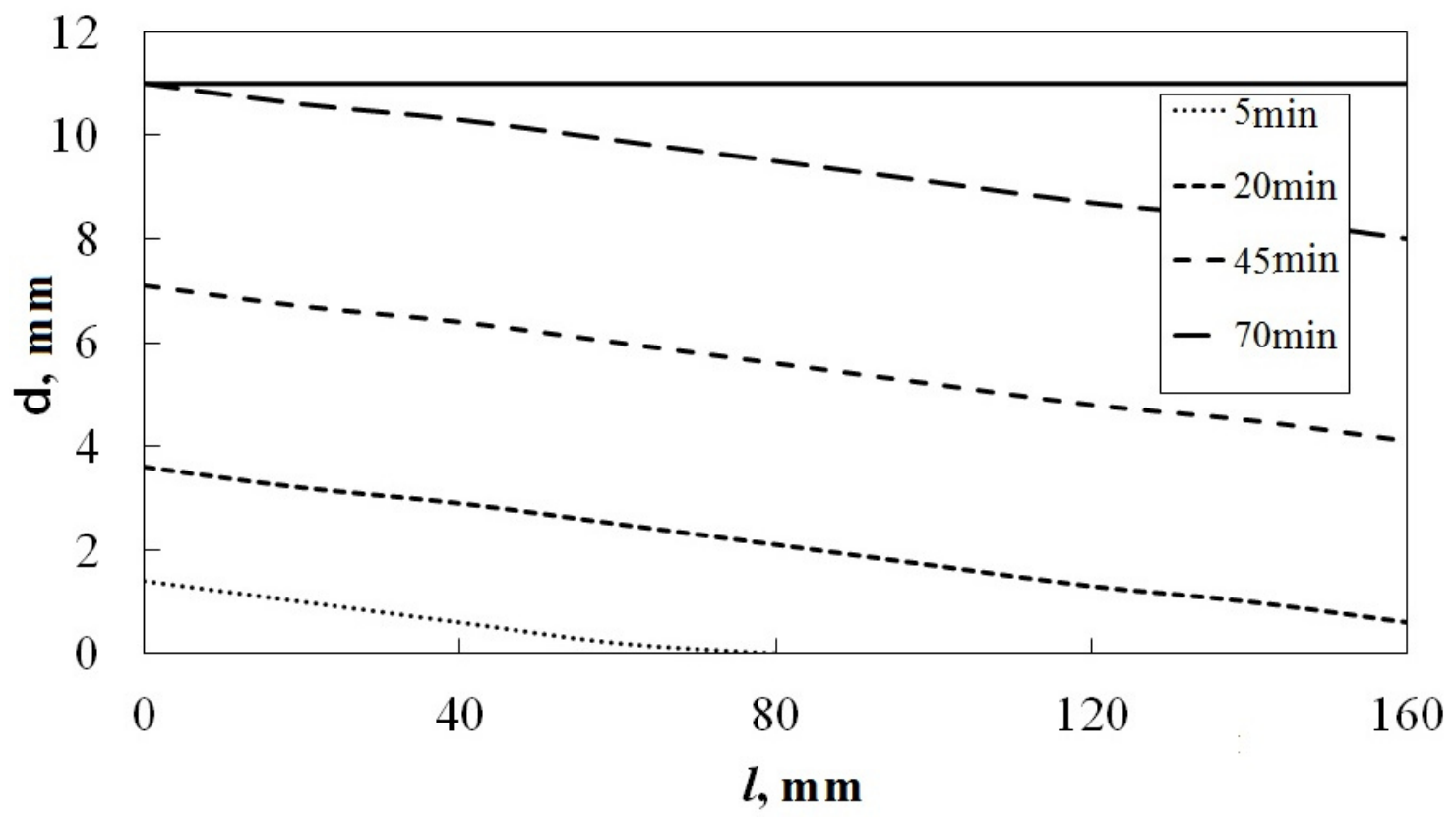

Figure 6. Change the thickness of the ice along the length of the evaporator tubes.

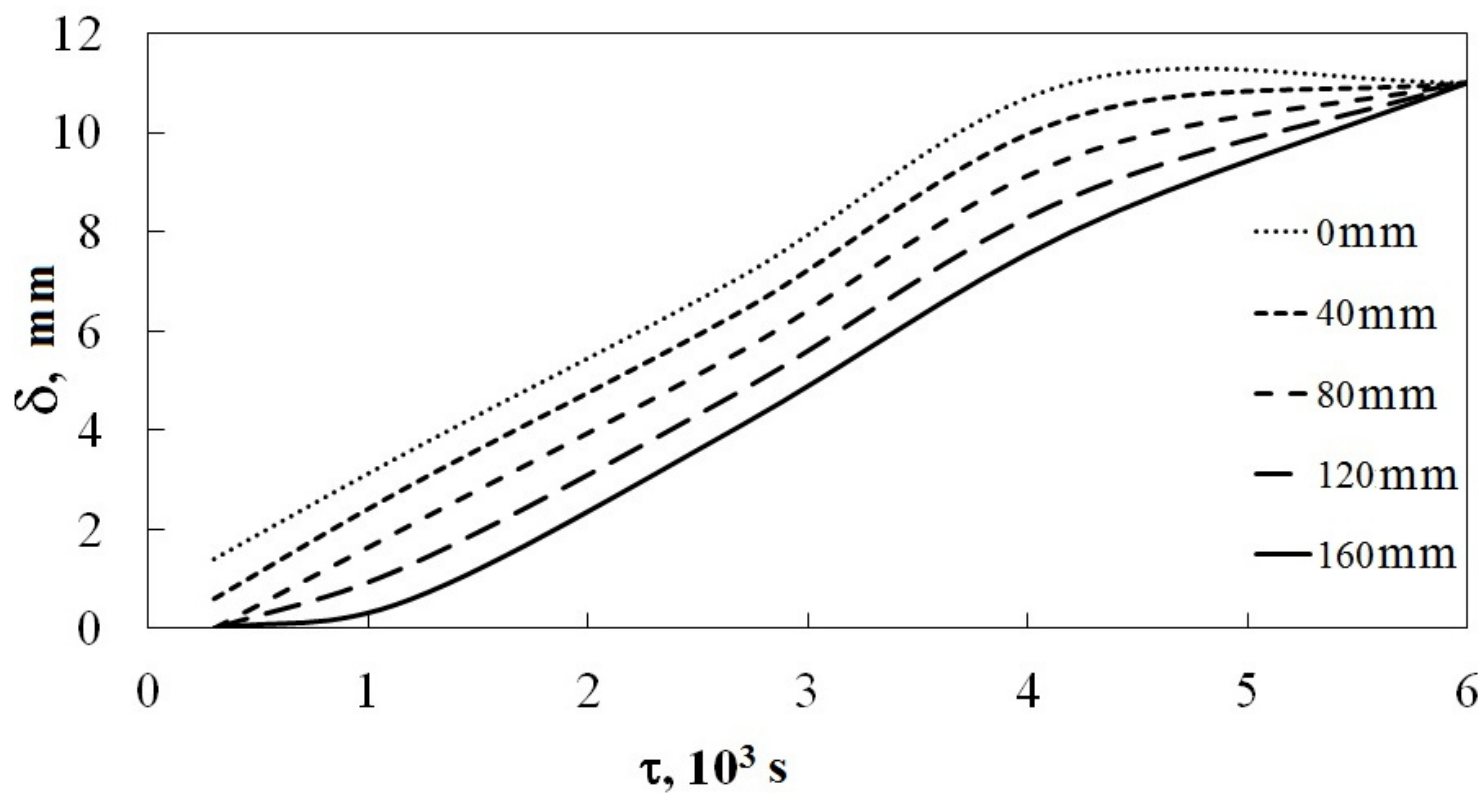

Figure 7. Changing ice thickness over time. 
Fig. 8 shows the values of the local temperature obtained as a result of experimental (in section $X=0,1$ м и $Y=0,1 \mathrm{м})$ and numerical studies. It can be seen that the change in temperature in time (characteristic points) meet the conditions of the generation of free-convection flow in this mode.

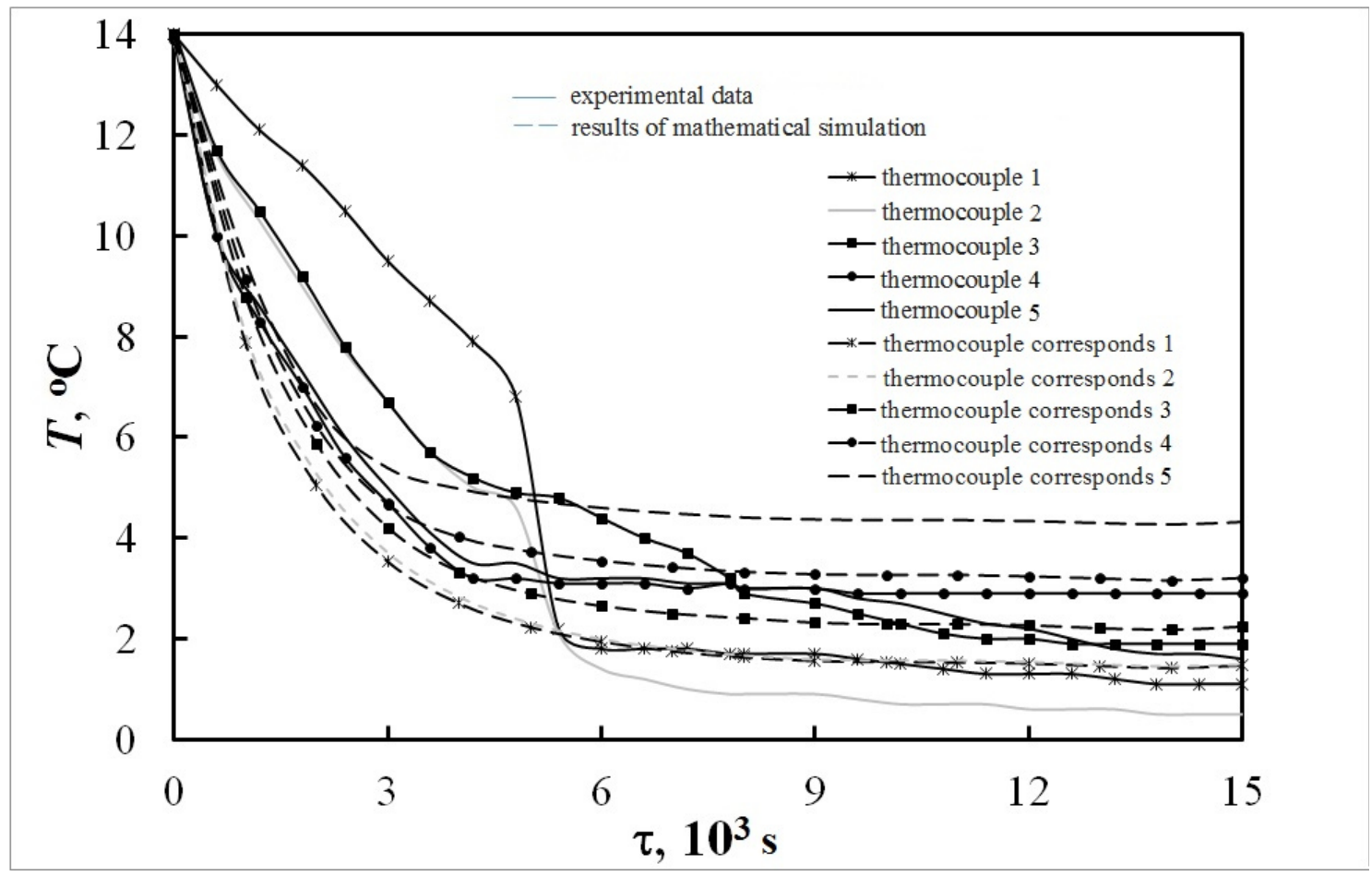

Figure 8. Change in water temperature at the time of low-grade heat source $14^{\circ} \mathrm{C}$

Intense convective flow, mixing of water masses in the region considered to be caused by a decrease in temperature at the initial time. At times more than 5000 seconds (Fig. 8) is noticeably sharp drop in temperature at several levels of the heat exchanger (thermocouples 1-4). This is due to the fact that in some areas the tank $T \approx 4^{0} \mathrm{C}$. At this value of $\mathrm{T}$ becomes the maximum density of water and changes the structure of convective flow. After that there is a slight decrease in temperature due to convection and a decrease in the intensity of the beginning of crystallization of the water on the surface of the heat exchanger.

\section{CONCLUSION}

The results of experimental studies illustrate the process of crystallization of the water on the surface of the heat exchanger. Crystallization has a significant effect on the thermal regime of the evaporator of the heat pump. Forming a layer of ice on the evaporator leads to uneven temperature distribution over the volume of the liquid in the chamber and significantly reduce the intensity of heat transfer in the heat exchanger. The results allow for the selection of the operating mode of HP in full or partial freezing of the evaporator. 
The work was realized within the research state assignment "Science" №13.1339.2014/K (Code of Federal Target Scientific and Technical Program 2.1410.2014).

\section{References}

1. Vezirshvili O.Sh., Meladze N.V., Jenergosberegajushhie teplonasosnye sistemy teplo- i hladosnabzhenija. M.: Izdatel'stvo Moskovskogo universiteta, 1994. - $106 \mathrm{~s}$.

2. Kuznetsov G.V., Sheremet M.A., Two-dimensional problem of natural convection in a rectangular domain with local heating and heat-conducting boundaries of finite thickness, Fluid Dynamics, 41 (2006), 6, pp. 881-890.

3. Kuznetsov G.V., Sheremet M.A., Conjugate natural convection in an enclosure with a heat source of constant heat transfer rate, International Journal of Heat and Mass Transfer, 54 (2011), 1-3, pp. 260-268.

4. Chamkha Ali J., Conduction-combined forced and natural convection in a lid-driven parallelogram-shaped enclosure divided by a solid partition, Progress in Computational Fluid Dynamics, 12 (2012), 5, pp. 309-321.

5. Kuznetsov G.V., Kurilenko N.I., Maksimov V.I., Mamontov G.Ya., Nagornova T.A., Heat transfer under heating of a local region of a large production area by gas infrared radiators, Journal of Engineering Physics and Thermophysics, 86 (2013), 3, pp. 519-524.

6. Maksimov V.I., Nagornova T.A., Influence of heatsink from upper boundary on the industrial premises thermal conditions at gas infrared emitter operation, EPJ Web of Conferences, 76 (2014), Article number 01006.

7. Vysokomornaya O.V., Kuznetsov G.V., Strizhak P.A., Heat and mass transfer in the process of movement of water drops in a high-temperature gas medium, Journal of Engineering Physics and Thermophysics, 86 (2013), 1, pp. 62-68.

8. Frank-Kameneckij D.A., Diffuzija i teploperedacha v himicheskoj kinetike. - M.: Nauka, 1987. $-502 \mathrm{~s}$.

9. Strakhov V.L., Garashchenko A.N., Kuznetsov G.V., Rudzinskii V.P., Mathematical simulation of thermophysical and thermo chemical processes during combustion of intumescent fireprotective coatings, Combustion, Explosion and Shock Waves, 37 (2001), 2, pp. 178-186.

10. Glushkov D.O., Kuznetsov G.V., Strizhak P.A., Numerical simulation of solid-phase ignition of metallized condensed matter by a particle heated to a high temperature, Russian Journal of Physical Chemistry B, 5 (2011), 6, pp. 1000-1006

11. Kuznetsov G.V., Maksimov V.I., Sheremet M.A., Natural convection in a closed parallelepiped with a local energy source, Journal of Applied Mechanics and Technical Physics, 54 (2011), 4, pp. 588-595. 